\title{
Anisotropy of Fracture Strength and Fracture Toughness of Micro-Sized Single-Crystal Silicon
}

\author{
Taeko Ando* Member \\ Xueping Li* Non-member \\ Shigeki Nakao* Non-member \\ Takashi Kasai* Non-member \\ Mitsuhiro Shikida** Member \\ Kazuo Sato* Member
}

\begin{abstract}
This paper presents the dependence of fracture toughness, fracture strength, and fracture behavior, such as crack propagation, on the crystal orientation of single-crystal silicon. We conducted on-chip tensile testing to measure fracture strength and fracture toughness of single-crystal silicon films with (100) and (110) surface in the $<100>$ and $<110>$ loading direction. The loading direction had a significant effect on fracture toughness, which was $2.17 \mathrm{MPa} \sqrt{\mathrm{m}}$ in the $<100>$ direction and $1.27 \mathrm{MPa} \sqrt{\mathrm{m}}$ in $<110>$. However, the fracture strength varies with both loading direction and surface orientation. We observed a fracture specimen on which a (111) cleavage plane eventually appeared on any crystal types of the specimen.
\end{abstract}

Keywords : Single crystal silicon, cleavage, tensile test, strength, fracture toughness, crack propagation

\section{Introduction}

Mechanical properties of structural film constituting MEMS (Micro Electro Mechanical Systems) devices have effects on features and performances of the devices. However no bulk data is available to apply to the thin film properties of which are strongly dependent on fabricating process and film thickness. Moreover, the properties of films are more easily influenced by the environments such as temperature and the existence of water. For such variation of properties in different sizes, the requirements of characterization of micro-sized materials has been raised.

Single-crystal silicon is one of the most commonly used materials in MEMS as pressure sensors, accelerometers, and various micro actuators. Several workers evaluated the mechanical properties of single-crystal silicon films by conducting tensile tests that they developed independently ${ }^{(1)-(4)}$. Recent studies showed that the micro-sized silicon has larger strength ${ }^{(5)}$ and more flexibility ${ }^{(6)}$, and the brittle-to-ductile transition point is lower as the size is down to nano-scale ${ }^{(7)}$. We need to check the fracture toughness as well as the strength for the micro-sized silicon films. Numerous researchers measured the fracture toughness by conducting the indentention methods and bending test with notched specimen and the data is summarized in, for example, literature ${ }^{(8)}$. In contrast, only a few measurements in small scale has been taken, for example, by using millimeter-sized ${ }^{(9)}$, micro-sized ${ }^{(10)}$, and nanometer-sized specimen ${ }^{(11)}$.

From Griffith's theory ${ }^{(12)}$, brittle fracture occurres because of cleavage crack propagation over the range of the equilibrium state, where the potential energy of material balances the surface energy generated by crack propagation. The Griffith theory shows the

* Dept. of Micro-Nano Systems Engineering, Nagoya University, Furou-cho, Chikusa-ku, Nagoya 464-8603

** EcoTopia Science Institute, Nagoya University, Furou-cho, Chikusa-ku, Nagoya 464-8603 crack is easy to propagete on the plane with low surface energy, which is cleavage plane in solid. In the case of silicon, two main clevage planes are (111) plane which is the most often appeared surface at failure due to the lowest surface energy ${ }^{(13)}$ and (110) which is secondarily easy to generate. The relationship between the crystal orientation and the loading condition is one of the factors to dominate the fracture. In addition to the crystal orientaion, surface defects are significantly important to motivate study of the fracture behavior in silicon thin film which is possible to neglect the inner defects. Cracks originate at the local stress concentration caused by defects on the surface such as a flaw or a pit introduced during the fabrication processes.

In this study, we investigated the effect of crystallographic orientation on fracture strength and fracture toughness of micro-sized silicon films in relation to the tensile axis and surface orientation. We also observed fractured specimens to reveal the fracture behavior that fracture occurs at the deffect on sidewall and porpagetes on cleavage plane.

\section{Experiment}

2.1 Testing Method We used the on-chip tensile testing method mentioned above in this work for measuring the fracture stress and fracture toughness. Fig. 1 shows the device structure

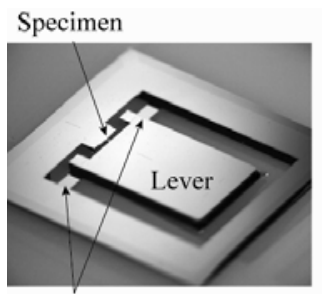

Torsion bars

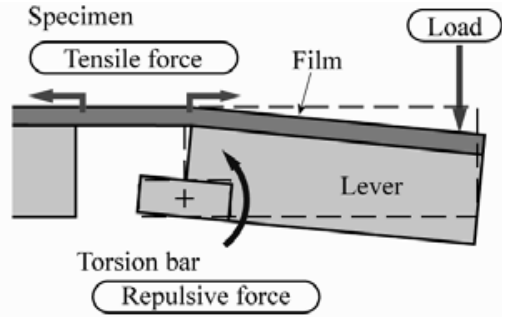

Repulsive force
Fig. 1. On-chip tensile testing device 
and the principle of this method. The device has a specimen and a loading system that is composed of torsion bars, a lever, and a frame on a single chip as shown in Fig. 1(a). When a load is applied to the lever, it rotates around the axis of the torsion bar and the specimen is stretched (Fig. 1(b)). The tensile force applied to the specimen can be calculated from the load applied to the lever by subtracting the repulsive force of the torsion bar that is independently measured after the specimen is broken. The details of our on-chip tensile testing method were previously presented (1)(10).

2.2 Specimen A schematic of the test specimen is shown in Fig. 2. We prepared two types of specimen "with notch" and "without notch" for measuring the fracture toughness and fracture strength, respectively. Both specimens were $100 \mu \mathrm{m}$ long, $45 \mu \mathrm{m}$ wide, and about $4 \mu \mathrm{m}$ thick. The V-shaped notches were introduced by using a focused ion beam (FIB) on a single side of the specimen with an opening angle of $22.6^{\circ}$ and its tip radius of about $30 \mathrm{~nm}$. The notch length were $0.7,0.75,0.8,1.0 \mu \mathrm{m}$. In order to examine the effect of crystallographic orientation on fracture properties in the silicon crystal the top surfaces of the specimen have two orientations, (100) and (110), and the tensile axes are aligned with $<100>$ and $<110>$.

We prepared two kinds of SOI wafers to fabricate the test chip. Silicon (100) and (110), as a top layer of SOI were aligned to a (100) silicon substrate in order that the orientaion flat of the substrate corresponds to the loading direction. The fabrication process of the test chip was composed of the three etching steps described in reference ${ }^{(1)}$. In the fabricated specimnen, the sidewalls were slightly roughened due to the reactive ion etching (RIE) process compared to the top and rear plane which were flat surface originally from the silicon wafer.

\section{Results and Discussion}

We used (100) and (110) silicon cut out of p-type CZ wafers as a specimen. All specimens of single crystal silicon film suffered brittle fractures without any plastic deformation in the tensile test. The results of the tensile test for the silicon film "with" and "without" notch are summarized in Table 1 . The average value and

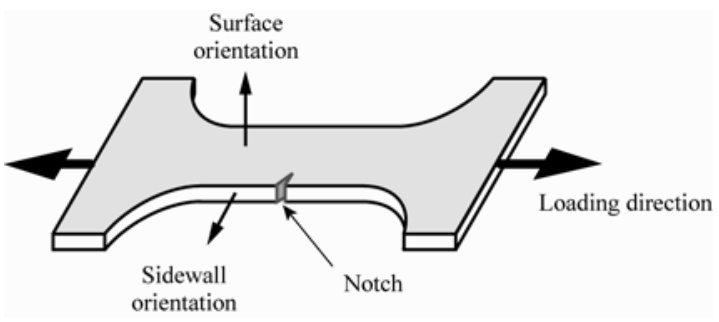

Fig. 2. Schematic view of test specimen standard deviation are listed for the fracture toughness and fracture strength.

3.1 Fracture Toughness We calculated the fracture toughness $K_{I C}$, in mode I failure, from the fracture stress and notch dimensions. The mode I stress intensity factor $K_{I}$ for single-edge notched specimen under tension is expressed by the following equation:

$$
K_{I}=\sigma \sqrt{\pi a} F(a / W)
$$

where $W$ and $a$ are the specimen width and crack depth, respectively. $F$ is the correction factor depending on crack shape and geometric boundaries given as follows:

$$
\begin{aligned}
F(a / W)= & 1.12-0.231(a / W) \\
& +10.55(a / W)^{2}-21.72(a / W)^{3}+30.39(a / W)^{4}
\end{aligned}
$$

In this study, we regarded the notch length as a crack depth in the above calculation. During the notch fabrication in the specimen, gallium ion with high energy at $30 \mathrm{keV}$ introduced crystal damages in the surface and sidewall such as generating defects and dislocations, mixing the gallium ion, and amorphisation. It is reported that the thickness of the damage layer is $20 \sim 40 \mathrm{~nm}$ at $30 \mathrm{keV}$ acceleration energy for commercially available FIB equipment ${ }^{(14)-(15)}$.

In Fig. 3, the measured fracture toughness is plotted as a function of the introduced notch length from $0.7 \mu \mathrm{m}$ to $1.0 \mu \mathrm{m}$. For each sample having different combination of surface orientation and loading direction, the fracture toughness had no change with increasing the notch length. It suggests that the fracture toughenss value is independent on the notch dimension in the range of $0.7-1.0 \mu \mathrm{m}$ notch length which is quite small comparing to the specimen dimension. Moreover, Fig. 3 shows that the fracture toughness values are plotted in the equivalent

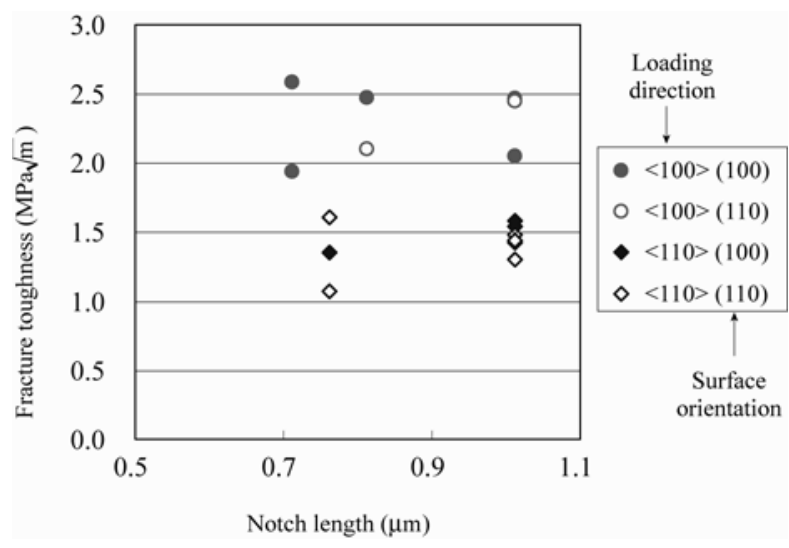

Fig. 3. Fracture toughness plotted as a function of

\begin{tabular}{|c|c|c|c|c|c|c|c|c|}
\hline \multirow[b]{2}{*}{$\begin{array}{l}\text { Loading } \\
\text { direction }\end{array}$} & \multirow{2}{*}{$\begin{array}{c}\text { Surface } \\
\text { orientation }\end{array}$} & \multirow{2}{*}{$\begin{array}{c}\text { Sidewall } \\
\text { orientation }\end{array}$} & \multicolumn{2}{|c|}{ With notch } & \multirow{2}{*}{$\begin{array}{c}\text { Surface } \\
\text { energy of } \\
\text { crystal } \\
\text { plane }(13) \\
\left(\mathrm{J} / \mathrm{m}^{2}\right)\end{array}$} & \multicolumn{3}{|c|}{ Without notch } \\
\hline & & & $\begin{array}{l}\text { Number } \\
\text { of tests }\end{array}$ & $\begin{array}{l}\text { Fracture toughness } \\
\qquad(\mathrm{MPa} \sqrt{m})\end{array}$ & & $\begin{array}{l}\text { Number } \\
\text { of tests }\end{array}$ & $\begin{array}{l}\text { Fracture strength } \\
\qquad(\mathrm{GPa})\end{array}$ & $\begin{array}{r}\text { Weibull } \\
\text { modulus }\end{array}$ \\
\hline \multirow{2}{*}{$<100>$} & $(100)$ & $(100)$ & 5 & $2.16 \pm 0.26$ & \multirow{2}{*}{$\begin{array}{l}2.13 \\
(100)\end{array}$} & 7 & $5.36 \pm 1.26$ & 2.51 \\
\hline & (110) & (110) & 3 & $2.19 \pm 0.17$ & & 7 & $5.51 \pm 1.46$ & 1.98 \\
\hline \multirow{2}{*}{$<110>$} & $(100)$ & (110) & 4 & $1.33 \pm 0.09$ & \multirow{2}{*}{$\begin{array}{c}1.51 \\
(110)\end{array}$} & 10 & $4.82 \pm 0.91$ & 4.30 \\
\hline & (110) & $(100)$ & 5 & $1.23 \pm 0.18$ & & 5 & $6.91 \pm 0.43$ & 11.82 \\
\hline
\end{tabular}
notch length

Table 1. Measured fracture properties of single crystal silicon 
range with the same loading direction, but are independent on the surface orientaion. Table 1 shows that the mean values of fracture toughness obtained from different surface orientations agreed with each other under the same loading condition. The measured mean values were $2.17 \mathrm{MPa} \sqrt{\mathrm{m}}$ in the $<100>$ direction and 1.27 $\mathrm{MPa} \sqrt{\mathrm{m}}$ in the $<110>$. This suggested that the fracture toughness of single crystal silicon is an inherent value determined by the loading direction, but not related to the surface orientation and notch length. In a simple consideration, the fracture toughness is related to the elastic constant, surface energy, and specimen geometory according to the Griffith's theory. In this test, the difference of the values such as elastic constant and surface energy due to crystallographic anisotropy resulted in the dependency of the fracture toughness on the loading condition.

3.2 Fracture Stress The measured fracture strength of silicon without notch is plotted in Fig. 4 as a function of both loading direction and surface orientation. The averaged values are listed in Table 1. Compared with the result of fracture toughness, the fracture strength vary with both loading direction and surface orientation, especially the results under $<110>$ loading were greatly different between two surface orientations. Moreover, the measured values widely ranged from a half of the average to a value slightly above the average. Some specimens tended to be extremely weak and they reduced the average value. The Weibull plots of the tensile strength measurements for the four types of single crystal silicon films are shown in Fig. 5.

The effect of surface orientation on the fracture stress implies that the magnitude of fracture strength of single crystal silicon films is not simply explained by the brittle fracture to break the bonds between atoms. The reason for this surface orientation effect may be from another contributions such as the fracturing manner described in section 3.3 and the condition of the initial fracture point that have an influence to the fracture strength. The stress at the initial fracture point is locally higher than the mean stress in the specimen. The local stress concentration are likely caused at the intersection of straighe line and curvature in the specimen profile and the area of the occurrence of the defect.

From the view of the fracture toughness, it is determined from the stress, crack depth, and specimen geometories. In the case of the $<110>$ loaded specimen, the measured fracture toughnesses were same values for both (100) and (110) specimens, so that it is assumed that the difference of the fracture strength comes from the differenth of the crack depth, in other words the magnitude of the stress concentration. By the SEM observation of the specimen

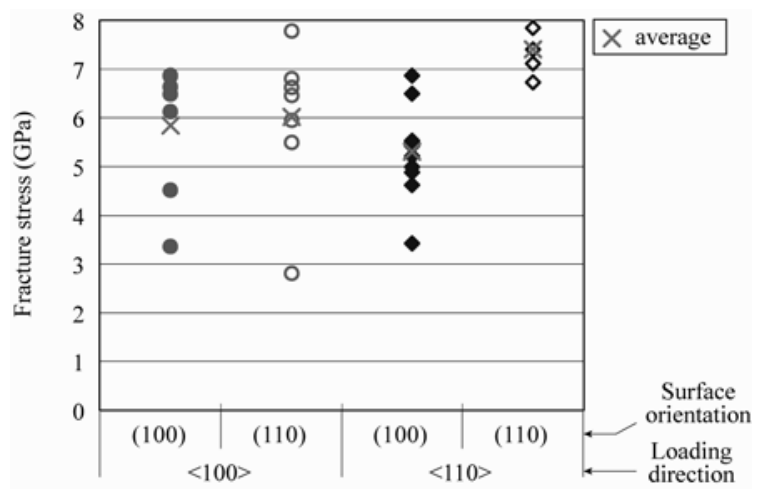

Fig. 4. Comparison of fracture strength of surface orientation and loading direction

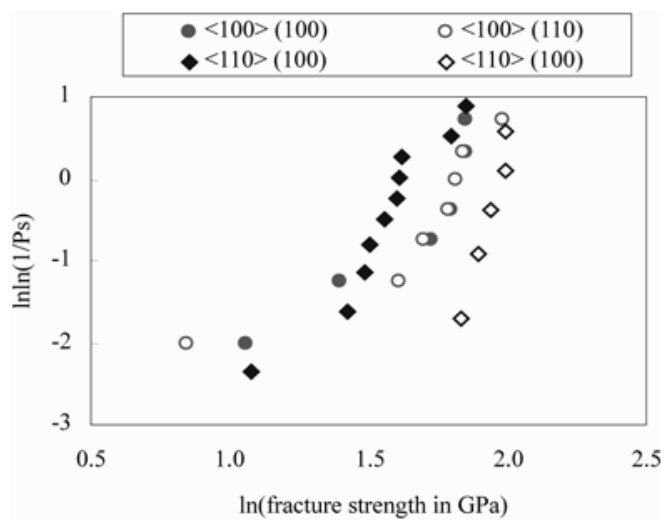

Fig. 5. Comparison of Weibull plots of fracture probability as a function of fracture strength for four types of tensile specimen of silicon

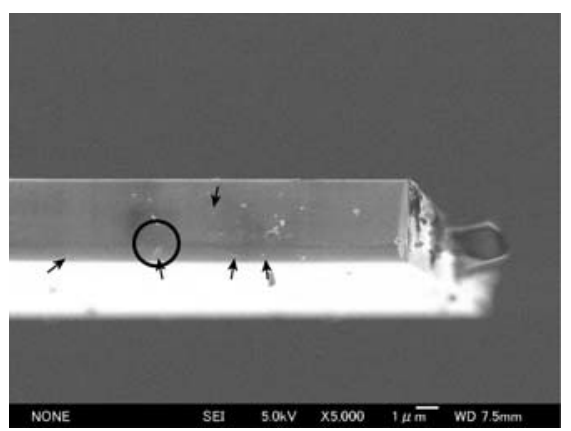

(a) (100) specimen with defects indicated by arrows on (110) sidewall

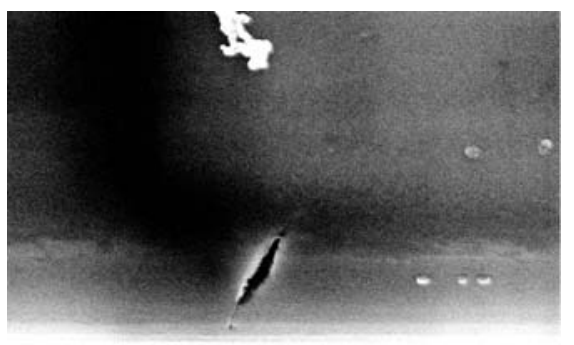

NONE

(b) The magnified view of the defects surrounded by circle in (a)

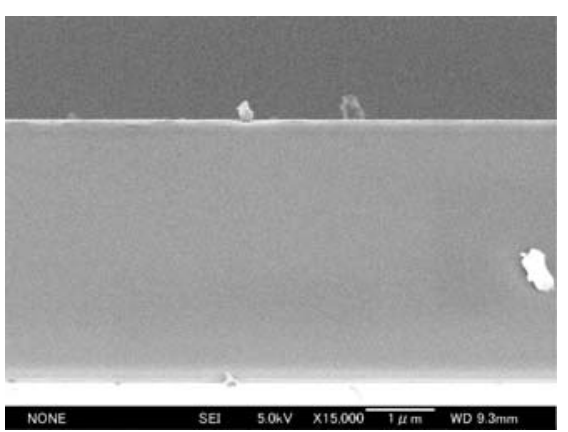

(c) (110) specimen with (100) sidewall

Fig. 6. SEM photographs of sidewall surface of the specimen for $<110>$ tensile direction 
sidewall after tensile test, the (100) specimen under $<110>$ loading have partly several flaws shown in Fig. 6(a) and (b), which may be introduced during the chip fabrication or under tensile loading and decrease the fracture strength. On the other hand, for the (110) surface specimen, the smooth surface without visible flaws were observed and may consequently increase the fracture strength shown in Fig. 6(c).

It is reported in the literature ${ }^{(16)}$ that the fracture strengths of single crystal silicon with a thickness of about $400 \mu \mathrm{m}$ under 3 -poing bending test are about $0.5 \mathrm{GPa}$ for both $<100>$ and $<110>$ longitudinal direciton. According to the literature ${ }^{(17)}$, the 3-point bending stengths of $400 \mu \mathrm{m}$ thick silicon are about $0.8 \mathrm{GPa}$ and $0.7 \mathrm{GPa}$ for $<100>$ and $<110>$ longitudinal direction, respectively. Compared to these values which are generally higer than those under tensile test when the specimen has the same dimension, the measured fracture strength in this study shows the higher values and larger scatter. The fracture origin in the micro-sized specimen caused by the stress concentration may be so small that the tensile strengths are widely ranged at higer value compared to the macro-sized specimen even bending strength.

3.3 Fracture Behavior The fracturing manner of single-crystal silicon was observed in the broken specimen obtained from the notched specimen. The specimen without notch broke and disappeared between expanding areas with curves at the end of the straight portion of the specimen due to impact at the fracture. The notched specimen decreased the fracture stress to below a quarter of that of the without notch specimen and remained whole after the tensile test. SEM photographs of the fractured specimen in each loading direction and surface orientation are shown in Fig. 7. The fracture occurred from the notch tip at first and then progressed in two different ways, moving straight (Fig. 7(c)) and branching in two directions symmetrically (Fig. 7(a), (b), and (d)). In Fig. 7(a), the braching angle between the first fracture progressing line and the appeared planes after branching is 45 degrees are tapered at 35 degrees. The fracture surfaces in Fig. 7(b) and (d) are vertical to the top surface and their branching angles are 55 and 35 degrees, respectively. All these inclined planes are (111) that is the first cleavage plane of single crystal silicon. However, in the case of $<110>$ loading on (100) specimen the crack propagated along one path perpendicular to the loading direction as is shown in Fig. $7(\mathrm{c})$. The fracture surface is (110), the second cleavage plane existing in the crystal structure of silicon.

Fig. 8 shows the fracture surface of $<110>$ loading specimen as observed with SEM. The (110) fracture surface that was obtained from the (100) specimen on a $<110>$ load (same as Fig. 6 (c)) was comprised of many narrow inclined planes alternating between acclivity and decline. The narrow planed start at the notch tip and passed over the fracture surface in the width direction. The orientation of both acclivity and decline planes was (111). The fracture surface actually appeared as a (111) cleavage plane in a micro view as if the second cleavage plane, (110), appeared in a macro view. We concluded that the fracture eventually occurred along a cleavage plane, (111), that was inclined for the loading direction in any pair of loading direction and surface orientation.

The (111) cleavage surface obtained from the other specimens was extremely smooth excepting the local area around the notch tip. Fig. 9 shows an example of the fracture surface observed

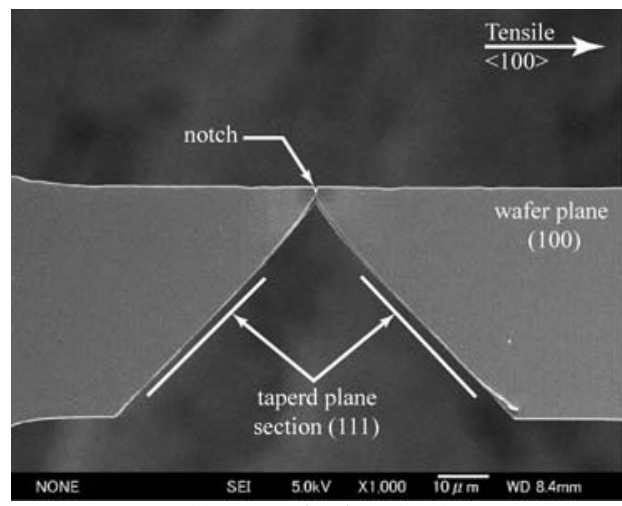

(a) $<100>$ load on $(100)$

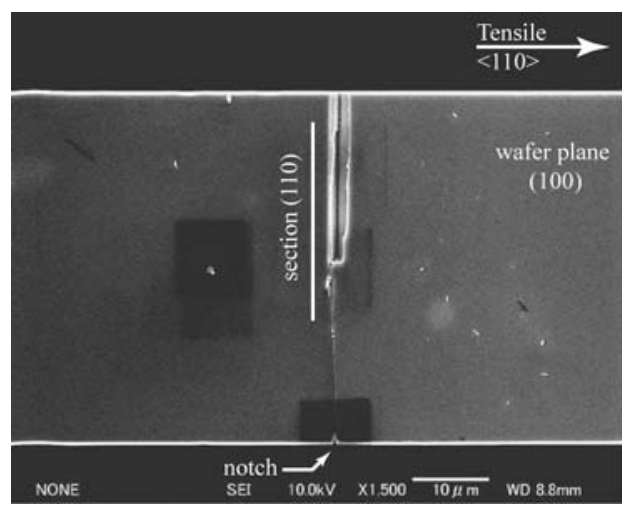

(c) $<110>$ load on $(100)$

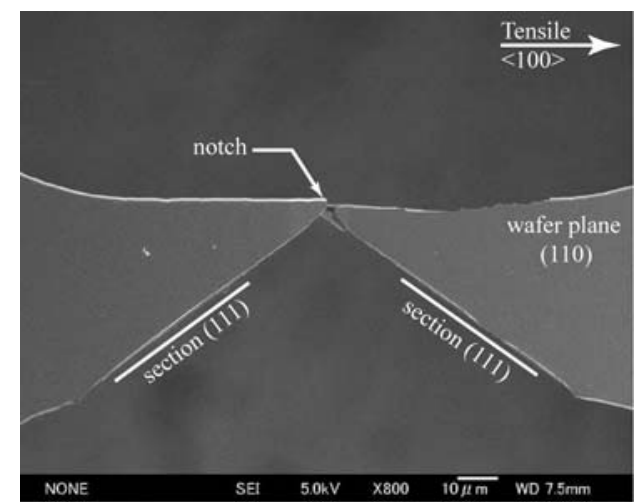

(b) $<100>$ load on $(110)$

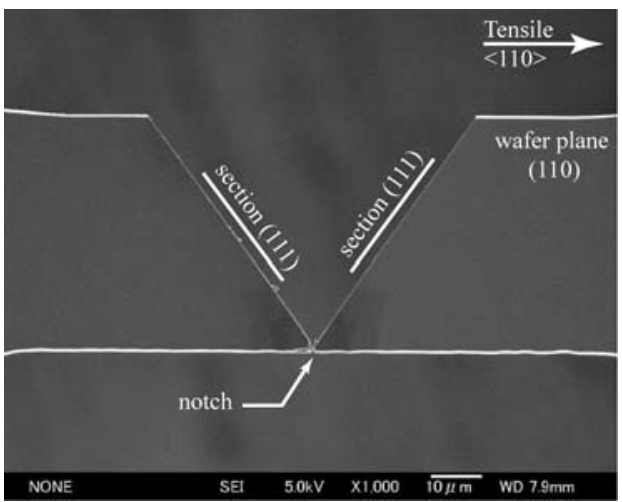

(d) $<110>$ load on (110)

Fig. 7. SEM photographs of fractured specimen with notch after tensile test 

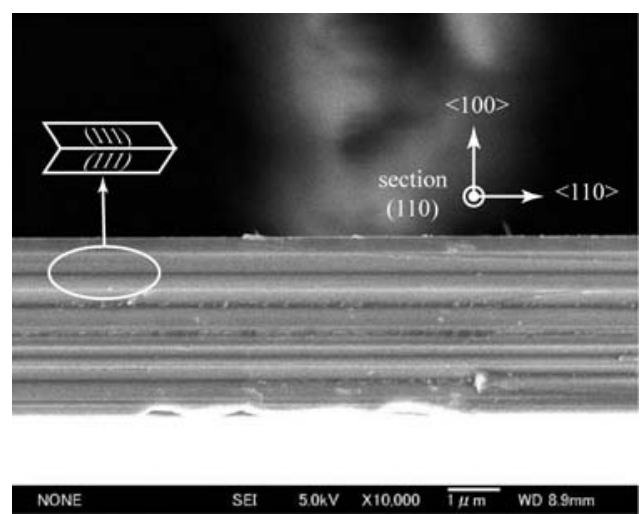

Fig. 8. SEM observation of fracture surface of (100) silicon after loading in $<110>$ direction

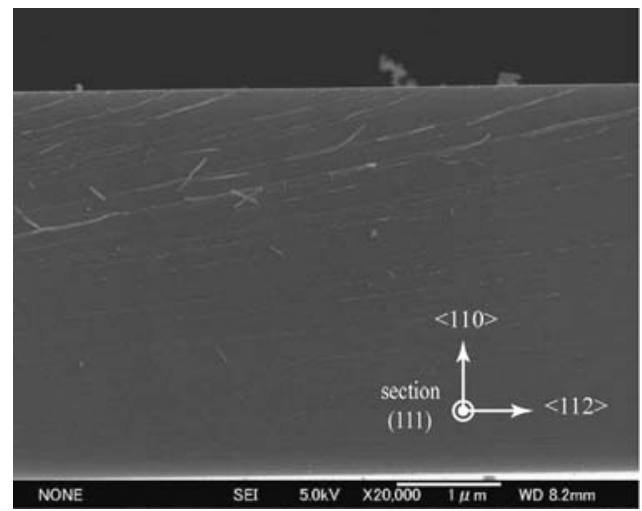

Fig. 9. SEM observation of (111) cleavage fracture surface of (110) silicon after loading in $<110>$ direction

vertically to the branching plane. The smooth plane was extended over the cross section with hairlines running parallel to each other. The hairlines showed on the edges of the steps on the (111) surface. This means that the fracture might have occurred from the notch to the other side with peeling layers between (111) planes.

\section{Conclusion}

We studied the dependence of fracture toughness, fracture strength, and fracture behavior such as crack propagation on crystal orientation. The measured values of fracture toughness were $2.17 \mathrm{MPa} \sqrt{\mathrm{m}}$ in the $<100>$ loading direction and 1.27 $\mathrm{MPa} \sqrt{\mathrm{m}}$ in $<110>$. We found that loading direction had a significant effect that led to a $58 \%$ decrease in the $<110>$ direction, and that surface orientation had no influence on the value. In the case of fracture strength, differences were observed in the value between both the loading direction and the surface orientation. One of the factor determinating the fracture strength was the existence of the deffect on the sidewall of the specimen. Two different types of fracture were observed not related to individual conditions. One branched two ways with a symmetric slope, and the other was along one path perpendicular to the tensile direction. However, fractures eventually occurred along a cleavage plane (111) in any crystal type of the silicon film specimen.

(Manuscript received Oct. 13, 2004, revised Mar. 10, 2005)

\section{References}

(1) K. Sato, T. Yoshioka, T. Ando, M. Shikida, and T. Kawabata : "Tensile testing of silicon film having different crystallographic orientations carried out on a silicon chip", Sensors and Actuators A: Physical, Vol.70, No.1-2, pp.148-153 (1998)

(2) T. Yi, L. Li and C. -J. Kim : "Microscale material testing of single crystalline silicon process effects on surface morphology and tensile strength", Sensors and Actuators A: Physical, Vol.83, No.1-3, pp.172-178 (2000)

(3) T. Tsuchiya, M. Hirata, N. Chiba, R. Udo, Y. Yoshitomi, T. Ando, K. Sato, K. Takashima, Y. Higo, Y. Saotome, H. Ogawa, and K. Ozaki : "Cross comparison of thin film tensile-testing method examined with single-crystal silicon, polysilicon, nickel, and titanium films", Proc. of MEMS2003, pp.666-669 (2003)

(4) F. F. Meroni and E. Mazza : "Fracture behaviour of single crystal silicon microstructures", Microsystem Technologies, Vol.10, pp.412-418 (2004)

(5) T. Ando, T. Yoshioka, M. Shikida, K. Sato, and T. Kawabata "Measurement of stress and strain of single-crystal-silicon thin film during on-chip tensile test", IEEJ Trans. S. M., Vol.119-E, No.2, pp.67-72, (1999-2)

(6) T. Lisby, S. A. Nikles, K. Najafi, O. Hansen, S. Bouwstra, and J. A. Branebjerg: "Mechanical characterization and design of flexible silicon microstructures", Journal of Microelectromechanical Syst., Vol.13, No.3, pp.452-464 (2004-3)

(7) T. Namazu, Y. Isono, and T. Tanaka : "Evaluation of size effect on mechanical properties of single crystal silicon by nanoscale bending test using AFM", Journal of Microelectromechanical Systems, Vol.9, No.4, pp.450-459 (2000-4)

(8) F. Ebrahimi and L. Kalwani : "Fracture anisotropy in silicon single crystal", Materials Science and Engineering, A268, pp.116-126 (1999)

(9) A. M. Fitzgerald, R. H. Dauskardt, and T. W. Kenny : "Fracture toughness and crack growth phenomena of plasma-etched single crystal silicon", Sensors and Actuators, Vol.83, pp.194-199 (2000)

(10) X. Li, T. Kasai, S. Nakao, H. Tanaka, T. Ando, M. Shikida, and K. Sato : "A Method for measuring the fracture toughness of micrometer-sized single crystal silicon by tensile test", Digest Tech. Papers Transducers'03 Conference, pp.444-447 (2003)

(11) S. Sundararajan and B. Bhushan : "Development of AFM-based techniques to measure mechanical properties of nanoscale structures", Sensors and Actuators A: Physical, Vol.101, pp.338-351 (2002)

(12) A. A. Griffith : "The phenomena of rupture and flow in solids", Philosophical Transactions of the Royal Society of London, Vol.221, pp.163-198 (1921)

(13) R. J. Jaccodine : "Surface energy of germanium and silicon", Journal of Electrochemical Society, Vol.110, No.6, pp.524-527 (1963)

(14) S. Lipp, L. Frey, C. Lehrer, B. Frank, E. Demm, and H. Ryssel "Investigations on the topology of structures milled and etched by focused ion beams", Journal of Vacuum Science and Technology B, Vol.14, No.6, pp.3996-3999 (1996)

(15) N. I. Kato, Y. Kohno, and H. Saka : "Side-wall damage in a transmission electron microscopy specimen of crystalline Si prepared by focused ion beam etching", Journal of Vacuum Science and Technology B, Vol.17, No.4, pp.1201-1204 (1999)

(16) K. Komai, K. Minoshima, H. Tawara, S. Inoue, and K. Sunako "Development of mechanical testing machine for microelements and fracture strength evaluation of single-crystalline silicon microelements", Trans. Japan Society of Mechanical Engineers A, Vol.60, No.569, pp.52-58 (1994)

(17) T. Ando : "On-chip tensile testing and fatigue testing of thin films", Ph.D. thesis, Nagoya University (2001)

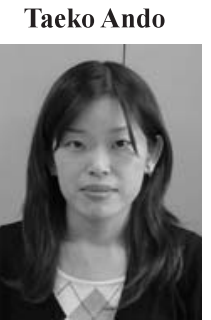

(Member) received her B.S. in aerospace engineering (1996) and M.S. and Ph.D in micro-system engineering (1998 and 2001) from Nagoya University, Japan. She has been a research associate of the Department of Micro-System Engineering at Nagoya University since 2001. Her research interests include evaluation of micro-mechanical structures. She is a member of the Institute of Electrical Engineers of Japan and the Japan Society for Precision Engineering. 


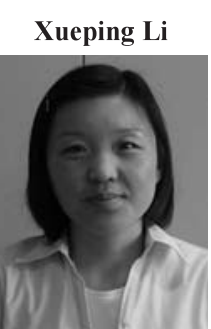

Shigeki Nakao

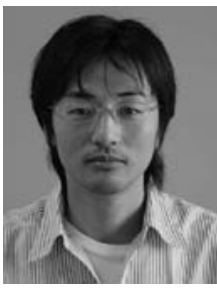

Takashi Kasai

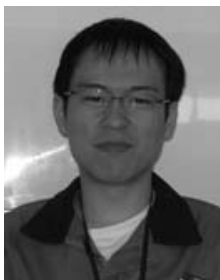

(Non-member) received her B.S. (1993) and M.S. (1996) degree from Nanjing University of Aeronautics and Astronautics (China). Currently, she is a $\mathrm{PhD}$ candidate in the Department of Micro System Engineering at Nagoya University, Japan. The subject of her thesis research is fracture properties in single crystal silicon micromechanical structures.

(Non-member) received B. S. degree in mechanical engineering from Nagoya University in 2003. He is currently doing M.S. degree at the Department of Micro System Engineering, Nagoya University. His research interests include evaluation of micro-mechanical structure properties.

(Non-member) received B.S. and M.S. degree in Micro System Engineering from Nagoya University in 2001 and 2003, respectively. He joined OMRON Corporation in 2003.
Mitsuhiro Shikida (Member) received his B.S. and M.S. degrees in

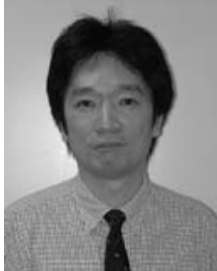
electrical engineering from Seikei University in 1988 and 1990. He received a Ph.D. from Nagoya University in 1998. From 1990 to 1995 , he worked at Hitachi, Ltd., Tokyo. In 1995, he joined Nagoya University as a research associate. He has been an assistant professor since 1998, and joined the Research Center for Advanced Waste and Emission Management (Currently, EcoTopia Science Institute) at Nagoya University in 2001. His research interests include microactuators, microfabrication, and micromechanical structures. Dr. Shikida is a member of the Institute of Electrical Engineers of Japan and the Japan Society of Mechanical Engineers.

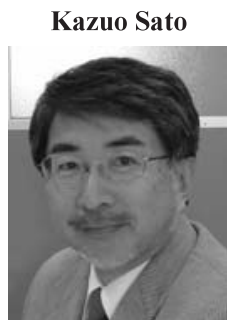

(Member) received B.S. degree in mechanical engineering from Yokohama National University in 1970 and Ph.D. from the University of Tokyo in 1982. In 1970, he joined Hitachi, Ltd., Tokyo. He has been studying micromachining technologies and their application since 1983, and has been a professor at Nagoya University since 1994 . Dr. Sato is a member of the Japan Society of Mechanical Engineers, the Japan Society for Precision Engineering, the Institute of Electrical Engineers of Japan, and the Japan Society for Technology of Plasticity. 